\title{
Controlling a quantum system via its boundary conditions
}

\author{
Christian Duffin ${ }^{1}$ and Arend G. Dijkstra ${ }^{1,2, a}$ \\ 1 The School of Physics and Astronomy, University of Leeds, Leeds LS29JT, UK \\ 2 The School of Chemistry, University of Leeds, Leeds LS29JT, UK
}

Received 12 July 2019 / Received in final form 9 September 2019

Published online 29 October 2019

(C) The Author(s) 2019. This article is published with open access at Springerlink.com

\begin{abstract}
We numerically study a particle in a box with moving walls. In the case where the walls are oscillating sinusoidally with a small amplitude, we show that states up to the fourth state can be populated with more than 80 percent population, while higher lying states can also be selectively excited. This work introduces a way of controlling quantum systems which does not rely on (dipole) selection rules.
\end{abstract}

\section{Introduction}

Reliably steering a quantum system from the ground state into a specific state is a well-known goal in quantum technology [1]. Techniques based on laser irradiation such as Rabi oscillations or stimulated Raman adiabatic passage (STIRAP) [2] are examples that have been designed for two- or three-level systems. In these methods, light directly interacts with one or multiple transitions in the quantum system. Transitions between the ground state and the desired state can be driven directly, or, intermediate states can be used. In the context of the Floquet theory for periodically driven systems, dipolar external forces have also been considered [3,4]. These techniques require either resonances with (dipole) allowed transitions or high-intensity external fields to be able to transfer a large amount of population.

Here, we introduce an alternative way of populating arbitrary states in a multi-level quantum system, which operates by varying the boundary conditions in time. Related to this idea, in the context of optical lattices, ac modulation of the lattice depth $[5,6]$, and a harmonic trap with large-amplitude fluctuations in the frequency [7] have been considered. In this letter, in which we focus on transferring population, we look at the well-known particle in a box system as an example. The boundary condition to be varied in this case is the length of the box, which is changed by moving one of the walls.

The particle in a box is a well-understood quantum system, and is largely used as a heuristic system in standard introductory textbooks $[8,9]$. But extending to the case of time-dependent boundaries, the literature is largely mathematically driven and because the system is no longer trivially solvable, this problem is still of current interest [10].

For any motion that is slow, the adiabatic approximation will suffice [11], in which the expansion coefficients $\left(C_{n}\right.$ defined later) of the system can be assumed to be time

\footnotetext{
a e-mail: a.g.dijkstra@leeds.ac.uk
}

independent. Because this assumption does not hold generally, exact solutions are limited to select cases. Analytical solutions of the time-dependent Schrödinger equation are known for constant wall velocity $[12-14]$ and for certain cases of the quantum harmonic oscillator, in which the angular frequency of the potential is parametrised as a specific function of the well width, $L$ [15-17]. Cooney has also proposed a means of deriving solutions for accelerating walls using extended transformation methods [18], although these only hold in the limit that the acceleration is slow. Employing a numerical approach [19], we are able to study arbitrary wall motion. Our aim in this paper was not to study the delicate mathematical issues related to moving walls, but to perform numerical simulations to study the behaviour of the particle.

\section{Model}

The usual particle in a one-dimensional box, as it is introduced in standard textbooks, has infinitely high walls on both sides of a box with length $L$, in which a particle with mass $m$ resides. Elementary solutions to the time-independent Schrödinger equation give the energy levels (eigenstates) for each integer quantum number $n$ as $E_{n}=\hbar^{2} \pi^{2} n^{2} / 2 m L^{2}$. The corresponding eigenfunctions are $u_{n}=\sqrt{2 / L} \sin n \pi x / L$, where $0<x<L$ is the coordinate. Any wave function $\psi$ can be expanded on the complete basis of these eigenfunctions, and evolves in time according to the time-dependent Schrödinger equation as

$$
\psi(x, t)=\sum_{n} C_{n} u_{n} \exp \left(-i E_{n} t / \hbar\right),
$$

where $C_{n}$ are the expansion coefficients determined from the initial condition.

The numerical solution for a system with a moving wall, described with a time-dependent box length $L(t)$, is 
obtained by allowing the expansion coefficients $C_{n}$ to be time dependent [19-21]. The details of the derivation are presented in reference [19]. Briefly, the ansatz wave function with time-dependent expansion coefficients is plugged into the time-dependent Schrödinger equation. Simplifying using the orthogonality of the eigenstates as well as their explicit form, one arrives at the final result as a set of coupled linear differential equations for the expansion coefficients

$$
\begin{aligned}
\dot{C}_{k}(t) & =\sum_{n} \frac{2(-1)^{k+n} k n}{\left(n^{2}-k^{2}\right)} \frac{\dot{L}(t)}{L(t)} C_{n}(t) \\
& \times \exp \left(\frac{-i L^{2}(t)}{\hbar}\left(E_{n}(t)-E_{k}(t)\right) \int_{0}^{t} \mathrm{~d} \tau \frac{1}{L^{2}(\tau)}\right) .
\end{aligned}
$$

In this equation, dots denote time derivatives, and the energies acquire a time dependence through the varying box length. The set of coupled equations is solved numerically using the Dormand-Prince algorithm as implemented in MATLAB [22]. This method provides a relatively simple way to study the particle in a box with moving walls. The brute force method of discretization of the spatial coordinate or analytical techniques [23] could also be applied.

As the initial condition of our simulations, we will assume that the system is prepared in the ground state. This choice could be easily generalized to superpositions of eigenstates, which would allow, for example, the study of quantum carpets [24-26].

In all our calculations, we will be working in natural units, taking the mass of the particle to be $m=1$, and $\hbar=1$. As a consequence, our time domain will be in units of $L_{0}^{2} \mathrm{~m} / \hbar$, where $L_{0}$ is the initial or average length of the box. For a proton in a $1 \mathrm{~nm}$ box, this time unit would correspond to $16 \mathrm{~ns}$.

\section{Constant velocity}

We validated our numerical approach by comparing timedependent populations of states with those obtained from known analytical solutions $[12,17,27]$ for a wall moving with a constant velocity $v$, that is, $L(t)=L_{0}+v t$. We briefly mention the results for a contracting box (negative $v$ ). When the box length becomes small, all state populations tend to a constant and the amplitude of oscillations decreases. The amount of population transferred from the ground state to other states increases with increasing speed of the wall motion. If the motion of the wall is sufficiently slow, little population is transferred, i.e. the system behaves adiabatically. For high enough speeds of the wall, it is possible to transfer most of the population out of the ground state, and higher lying states can be populated more than lower lying states. The ability of the wall to control the particle's momentum due to its nonlocalised nature has been referred to as the "Greenberger effect" $[17,27]$ and is a result of the delocalized nature of the wave function. Matzkin [10] has demonstrated that moving walls have no effect on the particle's state, as long as the wave function is vanishingly small at the position of

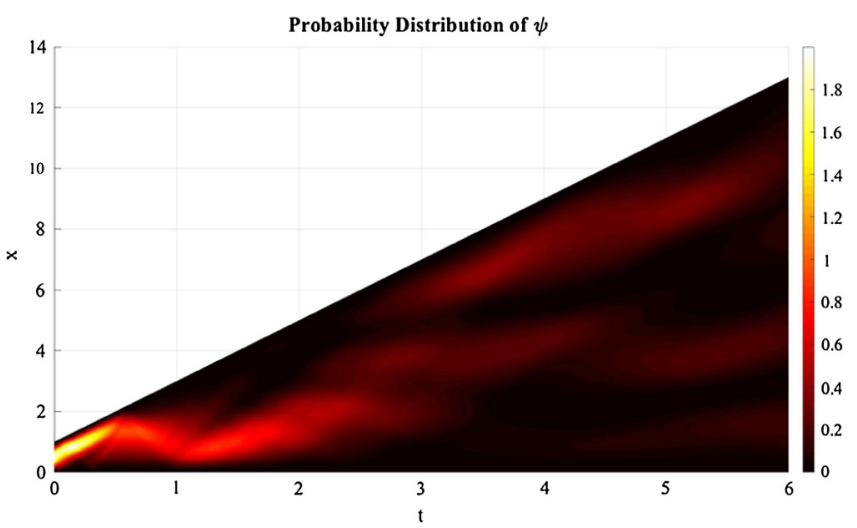

Fig. 1. Probability distribution for a uniformly expanding well with speed $v=2$ and $L_{0}=1$. The probability increases from black to red to yellow.

the wall and that therefore nonlocality is not present. The solution to the more general problem of why the whole wave function changes when only the part that touches the wall would be expected to be directly affected is still open, and has been described as a "conundrum" [21]. We remark that the Ehrenfest theorem implies that the expectation value of position is affected by the wall motion.

The states in a box with moving walls do not cross, nor do they exhibit avoided crossings. For a slowly varying system the adiabatic approximation is expected to be valid, and the particle mostly stays in the same time-dependent eigenstate. But, a uniformly expanding box is never eternally adiabatic. However slow the expansion, eventually the states will be so close together in energy that nonadiabatic effects become important. For a uniformly contracting box, the inverse principle is also true, which is to say that however fast the contraction, the states will eventually be sufficiently far from one another for the system to behave adiabatically.

There are several ways to visualize the particle in a box dynamics, which include plotting the eigenstate populations $\left|C_{n}\right|^{2}$, the expectations values of position $\langle\psi|x| \psi\rangle$, momentum $\langle\psi|p| \psi\rangle$, and kinetic energy $\left\langle\psi\left|p^{2} / 2 m\right| \psi\right\rangle$, or the probability distribution $|\psi(x, t)|^{2}$. The probability distribution for a particle in a linearly expanding box is shown in Figure 1 for a speed of $v=2$. From the increasing number of dark lines in this picture, it is clear that higher lying states are populated. Such effects are even more striking when the expansion speed is increased and are also reflected in expectation values of position and momentum.

\section{Acceleration}

While uniform motion has allowed us to investigate the limits of adiabaticity, an exponentially moving wall may be a better model for contraction. We will use as the equation for the wall length $L(t)=L_{0} \exp v t / L_{0}$. For appropriately chosen (negative) $v$, we can initialise the system into a certain state using non-adiabatic transitions and then force it into an adiabatic regime. Based on additional 


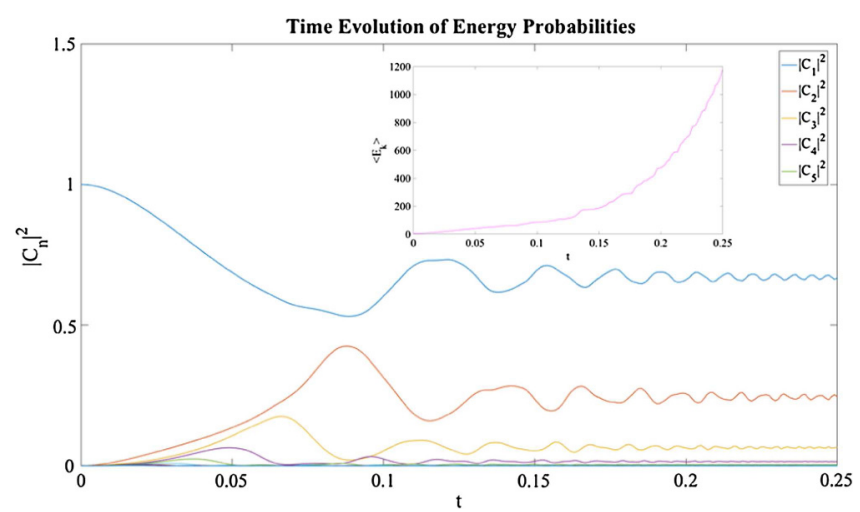

Fig. 2. State populations for an exponentially contracting well with $v=-9$ and $L_{0}=1$. The inset shows the kinetic energy.

simulations (results not shown) with $v=-3$ and $v=-20$, we believe that stable populations are generally obtained with an exponentially contracting wall. In this way, it becomes possible to prepare certain superpositions in a stable way. Results for the population dynamics are shown in Figure 2. This process could be used to accelerate a particle.

Oscillating wall-Our main results are for a sinusoidally moving wall. We set the length of the box as

$$
L(t)=L_{0}+v \sin \omega t,
$$

with angular frequency $\omega$ and amplitude $v$, as well as average box size $L_{0}$.

In Figure 3 we show the time dependence of the second and fourth states for a box driven with a frequency chosen in such a way that these states acquire the maximal possible population for the chosen driving amplitude. The figure shows that the excitation is selectively populating the desired state, with only small populations of other states. By doing this the system absorbs energy by increasing the kinetic energy of the particle. To prevent the population from going back to the ground state, as is the case for periodic driving, one could imagine more complex driving patterns to stabilize a desired state, for example, following periodic motion with exponential wall motion.

To investigate which driving frequency must be chosen to excite each state, we plot Figure 4. This figure shows the highest population of the first five particles in a box's eigenstates across a time domain of $t=0$ to $t=10$ as a function of the driving frequency $\omega$. In this figure, we observe many sharp resonances, which indicate optimal driving frequencies. Remarkably, there are peaks for each of the states, showing that each state can be driven to large population. The first four states all have maximum populations above 80 percent, confirming that they can be selectively excited. Although not shown here, we have also plotted a similar figure for even higher lying states, and we have confirmed that they can also be populated significantly through similar resonances (population larger than 0.3 for all states up to $n=10$ ).

We find that the resonance frequency needed to populate the second eigenstate is $\omega=14.7605$, close to the expected value of $E_{2}-E_{1}=3 \pi^{2} / 2=14.8044$. We attribute the small difference to the non-infinitesimal amplitude of the wall motion. Resonances to higher lying peaks cannot be explained with this simple argument. For completeness, we have also explored other values of the amplitude $v$ such as $v=0.04$ and $v=0.06$. As expected, we find similar resonances as in the case of $v=0.05$, but the maximum populations can be tuned by changing $v$.

At this point, we note that populating higher lying particles in a box's states with a dipolar force requires high intensities [4]. In contrast, our method produces large populations with small amplitude driving [28] of the wall. Indeed, we can populate the lowest 6 states with more than 60 percent population, and the lowest four states with more than 80 percent. This could lead to more efficient ways of populating such states.

To complete our investigation into sinusoidal motion, we have also considered motion with a low frequency [18] but a large amplitude. In this case, we find a similar interplay between adiabatic evolution and non-adiabatic mixing as expected from the exponentially moving wall.

\section{Experimental systems}

State selective excitation techniques such as STIRAP have many applications [2]. Implementation of our numerical findings could be attempted in optical lattices, optomechanical resonators $[29,30]$ or in nano-electro-mechanical systems [31]. Furthermore, acceleration by a moving wall could be used to inject kinetic energy into particles. Recently, a potential with the characteristics of a particle in a box has also been used as a model system for excess protons in water [32], which is known to be a highly dynamic system [33], and could therefore be modelled with our approach.

\section{Conclusion}

In conclusion, we have been able to use numerical techniques in order to investigate the nature of the particle in a box with moving walls in terms of the adiabatic and the non-adiabatic regimes. Through this we have demonstrated a mechanism by which the particle can be prepared in a stable state of tuneable energy, controlled by the speed of contraction. We have also described a method to selectively populate quantum states by driving the system's boundaries periodically with a small amplitude by tuning the frequency. In future work, it will be important to investigate decay processes that lead to losses from the desired state. Techniques such as STIRAP employ coherences with an intermediate state without significantly populating it, therefore making the technique insensitive to losses from this state. However, in our technique, no intermediate state is necessary because the model does not rely on dipole allowed transitions. Also, the particle in an infinitely deep potential well is an artificial model, and more realistic model potentials such 


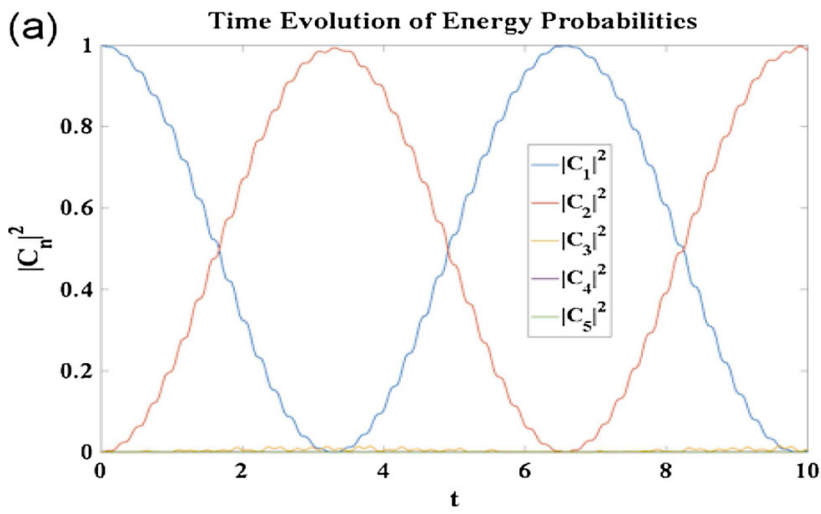

(b) 1.2 Probability Distribution of $\psi$

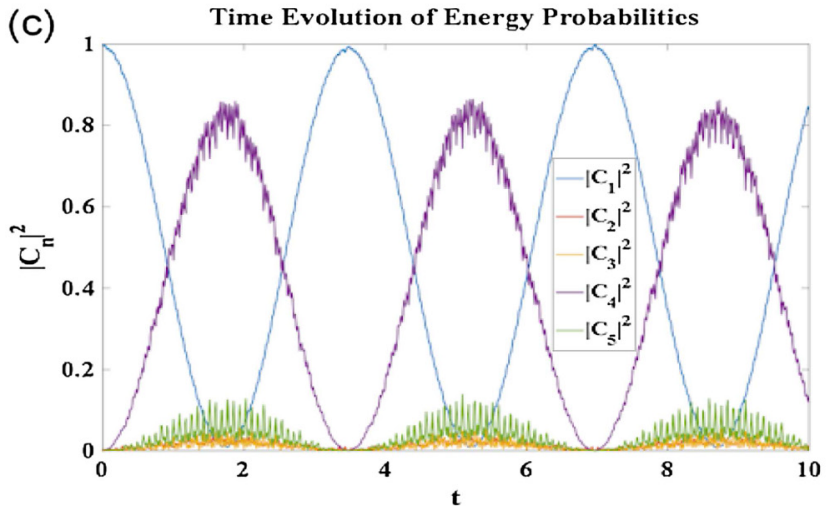

(d) 1.2

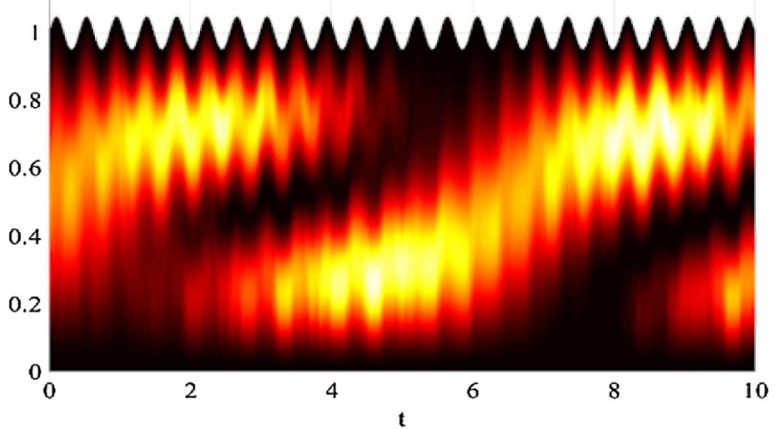

Probability Distribution of $\psi$

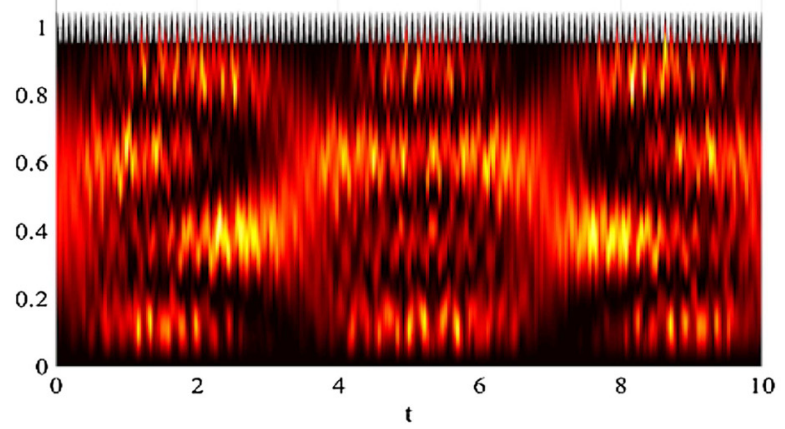

Fig. 3. (a) and (c) State populations and (b) and (d) probability distributions for a sinusoidally moving wall with frequency (a) and (b) 14.7605 and (c) and (d) 73.7048. and small amplitude $v / L_{0}=0.05$ and $L_{0}=1$. The frequency in the top (bottom) row is chosen to maximize the population in the second (fourth) eigenstate.

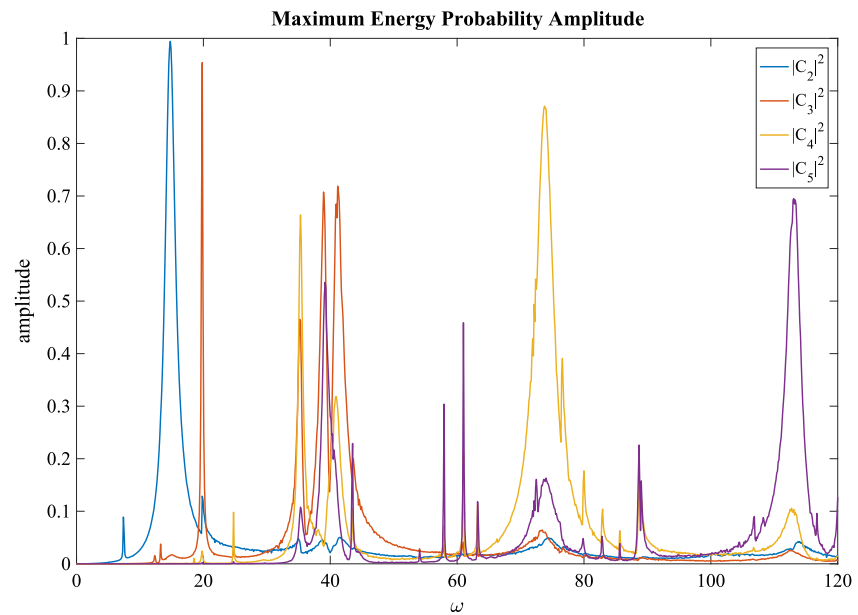

Fig. 4. Maximum populations of each state as a function of driving frequency for a sinusoidally moving wall with angular frequency $\omega, L_{0}=1$ and $v / L_{0}=0.05$. The sharp resonances allow selectively populating certain individual eigenstates.

as a finite well should be investigated. Extending this analysis to a stochastic regime of motion, such as Brownian motion [34], would be useful in describing the dynamics of excess proton in water systems. Finally, preparing the system in superposition states to simulate quantum carpets [24-26], it could be worth investigating how these patterns respond to the types of motion demonstrated in this work.
We thank Dr Zlatko Papić, Prof Jiannis Pachos and Dr Marcelo Miranda for helpful discussions.

\section{Author contribution statement}

AGD initiated the project. CD set up and performed simulations. Both authors discussed the results. AGD wrote the paper with input from all authors.

Open Access This is an open access article distributed under the terms of the Creative Commons Attribution License (http://creativecommons.org/licenses/by/4.0/), which permits unrestricted use, distribution, and reproduction in any medium, provided the original work is properly cited.

\section{References}

1. J.F. Haase, Z.Y. Wang, J. Casanova, M.B. Plenio, Phys. Rev. Lett. 121, 050402 (2018)

2. N.V. Vitanov, A.A. Rangelov, B.W. Shore, K. Bergmann, Rev. Mod. Phys. 89, 015006 (2017)

3. M. Bukov, S. Gopalakrishnan, M. Knap, E. Demler, Phys. Rev. Lett. 115, 205301 (2015)

4. M. Holthaus, J. Phys. B: At. Mol. Opt. Phys. 49, 013001 (2015) 
5. T. Stöferle, H. Moritz, Ch. Schori, M. Köhl, T. Esslinger, Phys. Rev. Lett. 92, 130403 (2004)

6. S.D. Sarkar, R. Sensarma, K. Sengupta, Phys. Rev. B 92, 174529 (2015)

7. H. Lignier, A. Zenesini, D. Ciampini, O. Morsch, E. Arimondo, S. Montangero, G. Pupillo, R. Fazio, Phys. Rev. A 79, 041601 (2009)

8. A. Rae, Quantum Physics: a Beginner's Guide (Oneworld Publications, 2005)

9. D.J. Griffiths, Introduction to Quantum Mechanics (Cambridge University Press, 2018)

10. A. Matzkin, J. Phys. A: Math. Theor. 51, 095303 (2018)

11. D.N. Pinder, Am. J. Phys. 58, 54 (1990)

12. S.W. Doescher, M.H. Rice, Am. J. Phys. 37, 1246 (1969)

13. V.V. Dodonov, V.I. Manko, D.E. Nikonov, Phys. Lett. A 162, 359 (1992)

14. V.V. Dodonov, A.B. Klimov, D.E. Nikonov, J. Math. Phys. 34, 3391 (1993)

15. A.J. Makowski, S.T. Dembinski, Phys. Lett. A 154, 217 (1991)

16. A.J. Makowski, J. Phys. A 25, 3419 (1992)

17. A.J. Makowski, P. Peplowski, Phys. Lett. A 163, 143 (1992)

18. K. Cooney, arXiv:1703.05282 (2017)

19. L. Tarr, Quantum systems with time-dependent boundaries, Ph.D. thesis, Reed College, 2005

20. A. Munier, J.R. Burgan, M. Felix, P. Peplowski, J. Math. Phys. 22, 1219 (1981)
21. A. Matzkin, S.V. Mousavi, M. Waegell, Phys. Lett. A 382 , $3347(2018)$

22. https://www . mathworks. com/help/matlab/ref/ode45. html

23. S. Di Martino, F. Anzà, P. Facchi, A. Kossakowski, G. Marmo, A. Messina, B. Militello, S. Pascazio, J. Phys. A: Math. Theor. 46, 365301 (2015)

24. M.V. Berry, J. Phys. A 29, 6617 (1996)

25. M.V. Berry, Phys. World 14, 39 (2001)

26. I. Marzoli, F. Saif, I. Bialynicki-Birula, O.M. Friesch, A.E. Kaplan, W.P. Schleich, Acta Phys. Slov. 48, 323 (1998)

27. D.M. Greenberger, Physica B 151, 374 (1988)

28. F. Anzà, A. Messina, B. Militello, Open Syst. Inf. Dyn. 26, 1950006 (2019)

29. N. Mann, M. Reza Bakhtiari, A. Pelster, M. Thorwart, Phys. Rev. Lett. 120, 063605 (2018)

30. H.K. Lau, A. Eisfeld, J.M. Rost, arXiv:1803.00150 (2018)

31. Y.I. Sohn, S. Meesala, B. Pingault, H.A. Atikian, J. Holzgrafe, M. Gündoğan, C. Stavrakas, M.J. Stanley, A. Sipahigil, J. Choi, M. Zhang, J.L. Pacheco, J. Abraham, E. Bielejec, M.D. Lukin, M. Atatüre, M. Lončar, Nat. Commun. 9, 2012 (2018)

32. F. Dahms, B.P Fingerhut, E.T.J. Nibbering, E. Pines, T. Elsaesser, Science 357, 491 (2017)

33. M. Thämer, L. De Marco, K. Ramasesha, A. Mandal, A. Tokmakoff, Science 350, 78 (2015)

34. T. Hida, Brownian Motion (Springer-Verlag, 1980) 